\title{
Editorial
}

http://dx.doi.org/10.14483/calj.v18n1.aa00

\section{English teaching in the elementary school: Some critical issues}

Although for the last 10 years Colombia has proposed a National Plan for Bilingualism in different stages, the education of young children in Colombian public and private schools in relation to English language learning evidences some critical issues regarding the preparation of future teachers, the professional development of in-service teachers, and the national standards for foreign language teaching.

The preparation of future teachers of English focuses mainly on delivering theoretical perspectives of English grammar, linguistics, traditional and decontextualized approaches to EFL teaching, second language acquisition theories, and research methods to mention some of the general content in English language education. The study carried out by Cardenas (2009) investigating the tendencies in language teacher education in Colombia reported that "transmission and language skill oriented models of teaching still exist at universities; educational perspectives that view the person as a social individual and promote a critical model of education are rarely found" (p.100). However, most programs fail to address the challenges and teaching realities that classrooms in public schools face every day. With a single and isolated experience in the practicum in elementary school, an absence of in-depth discussions about critical approaches to teaching in today's challenging times, and a lack of innovative and creative practices that articulate content from the disciplines in the curriculum, future teachers remain unprepared for teaching in classrooms full of children with diverse cultural and socioeconomic needs and literacies.

Similarly, national educational policies have mistakenly given elementary school teachers not necessarily certified as English teachers the responsibility of teaching English to elementary school children. This decision seems to be based on a limited vision that learning another language is learning isolated and decontextualized vocabulary or, administratively, making a simple curricular adaptation instead of having well qualified teachers to teach English to children.

In my professional opinion, this political decision not only delays the expected outcomes for English proficiency of school students to perform successfully as citizens, but it places elementary school teachers in an awkward and unethical professional position. By doing so, the government fails to provide opportunities for new English teachers who can be prepared and fully qualified to teach children. My qualms are that teachers who know how to teach children, but do not know how to teach English, will not be able to educate children as users of the language. In contrast, teachers learning about ELT methodology only from a theoretical perspective will not make them necessarily better teachers of the language.

Furthermore, if the Colombian government were to implement a coherent linguistic policy for foreign language learning to achieve the goals of the National Bilingualism Plan, it would have to offer quality EFL education to children from early elementary grades to 11 th grade. Putting in place a strong educational initiative for public schools would require hiring a good number of English teachers who are professionally qualified to teach in the elementary grades. It is 
equally important to take into consideration the immediate need for professional development for in-service teachers. Practicing teachers would require qualifying their practice through more social and culturally contextualized pedagogies of EFL that lead to innovative teaching using their educational realities and concentrating less on knowing about decontextualized theories. Teachers currently teaching in the secondary grades would need to renew their pedagogical practices to respond to the new demands of more competent students in English whose English would have been learned in the five previous elementary grades.

An example of a content based approach to teaching EFL to young learners is presented in this issue in the article titled "It's the same world through different eyes": a CLIL project for young EFL learners" by Eleni Korosidou and Eleni Griva. The authors define CLIL as a dualfocused educational approach which aims at education through construction, rather than instruction. Thus, their pedagogical innovation focused equally on EFL (English as a foreign language) and content development. In their study, students were provided with opportunities to express themselves verbally and nonverbally, and to participate in a variety of creative activities in a multimodal teaching context. The findings of their project evidenced students' improvement regarding both their receptive and productive skills in the target language, and the development of children's citizenship awareness, and their sensitivity towards diversity.

Lastly, the Colombian national standards for foreign language learning - in contrast with the standards for any other discipline-display a very limited range of linguistic competences and insist on focusing on the grammar of the language. This emphasis on learning about the language and not the language itself poorly connects with the life and realities of the learners who need to use English to fully participate as critical citizens.

\section{References}

Cárdenas, R. (2009). Tendencias globales y locales en la formación de docentes de lenguas extranjeras. Íkala, revista de lenguaje y cultura, 14(22), 71-105.

Coyle, D., Hood, P., \& Marsh, D. (2010). CLIL: Content and language integrated learning. Cambridge: Cambridge University Press.

Korosidou E., \& Griva, E. (2016). "It's the same world through different eyes": A content and language integrated learning project for young EFL learners. Colombian Applied Linguistics Journal, 18(1), pp 116-132.

Amparo Clavijo Olarte PhD

Editor 


\section{Editorial}

\section{La enseñanza del inglés en la escuela primaria: algunos problemas críticos}

Aunque durante los últimos 10 años Colombia ha propuesto un Plan Nacional de Bilingüismo en diferentes etapas, la educación de los niños pequeños en las escuelas públicas y privadas de Colombia en relación con el aprendizaje del Inglés pone de manifiesto algunos problemas críticos respecto a la preparación de los futuros docentes, el desarrollo profesional de maestros en ejercicio, y los estándares nacionales para la enseñanza de lenguas extranjeras.

La preparación de los futuros profesores de Inglés se centra principalmente en proveer perspectivas teóricas de la gramática del Inglés, la lingüística, los enfoques tradicionales y descontextualizados de la enseñanza del inglés como lengua extranjera, las teorías de adquisición de un segundo idioma, y métodos de investigación para mencionar algunos de los contenidos de la enseñanza del idioma Inglés en general. El estudio realizado por Cárdenas (2009), la investigación de las tendencias en la formación del profesorado de idiomas en Colombia, informó que "los modelos de transmisión de conocimientos orientados al desarrollo de habilidades de lenguaje aún existen en las universidades; las perspectivas educativas que consideran a la persona como un individuo social y promueven un modelo crítico de la educación rara vez se encuentran"(p.100). Sin embargo, la mayoría de los programas no tienen en cuenta los retos y realidades de enseñanza que las aulas de las escuelas públicas enfrentan cada día. Con una sola experiencia en la práctica en la escuela primaria, la ausencia de un debate a profundidad sobre enfoques críticos de la enseñanza en un momento difícil, como el actual, y la falta de prácticas innovadoras y creativas que articulen el contenido de las disciplinas del plan de estudios, los futuros maestros siguen sin estar preparados para la enseñanza en aulas llenas de niños con diversas necesidades, socioeconómicas y culturales y con uso de nuevas literacidades.

Del mismo modo, las políticas educativas nacionales le han dado, por error, a los docentes la responsabilidad de enseñar inglés a niños de primaria, sin estar necesariamente certificados como profesores de inglés. Esta decisión parece estar basada en una visión limitada de que el aprendizaje de otro idioma es el aprendizaje de vocabulario aislado y descontextualizado o, administrativamente, la realización de una adaptación curricular sencilla en lugar de tener maestros bien calificados para enseñarles inglés a los niños. En mi opinión profesional, esta decisión política no sólo retrasa los resultados esperados en cuanto al dominio del idioma por parte de los estudiantes para alcanzar metas como ciudadanos, sino que pone a los maestros en una posición incómoda y profesionalmente poco ética. Al hacer esto, el Gobierno no les proporciona a los profesores de inglés nuevas oportunidades para prepararse y estar totalmente calificados para enseñarles a los niños. Mi preocupación es que los maestros que saben cómo enseñar, pero no saben cómo enseñar inglés, no serán capaces de educar a los niños como usuarios de la lengua. Por el contrario, los maestros que desde la teoría estén aprendiendo acerca de la metodología de la enseñanza de inglés como lengua extranjera no serán, necesariamente, mejores maestros de la lengua.

Por otra parte, si el Gobierno de Colombia fuera a implementar una política lingüística coherente para el aprendizaje de lenguas extranjeras para cumplir los objetivos del Plan Nacional de Bilingüismo, se tendría que ofrecer una educación de inglés como lengua extranjera de calidad a los niños desde su infancia en grado primero hasta grado once. Poner en marcha una iniciativa educativa sólida para las escuelas públicas requeriría la contratación de un buen número de profesores de inglés que estén profesionalmente calificados para 
enseñar en primaria. De igual manera, es fundamental tener en cuenta la necesidad inmediata de desarrollo profesional para los maestros en servicio. Los profesores en ejercicio requerirían calificar su práctica a través de pedagogías más sociales y culturalmente contextualizadas del inglés como lengua extranjera que conduzcan a una enseñanza innovadora, utilizando sus realidades educativas y concentrándose menos en las teorías descontextualizadas. Los maestros que están enseñando actualmente en secundaria tendrían que renovar sus prácticas pedagógicas para responder a las nuevas demandas de estudiantes más competentes en inglés cuyo inglés habría sido aprendido en los cinco grados de primaria anteriores.

Un ejemplo de un enfoque basado en el contenido de la enseñanza del inglés como lengua extranjera a jóvenes aprendices es presentado en este número en el artículo "El mundo visto desde miradas diferentes": un proyecto que integra contenido y lenguaje para aprendices de inglés como lengua extranjera en primaria, de Eleni Korosidou y Eleni Griva. Las autoras definen CLIL como un método educativo de doble enfoque, que tiene por objeto la educación a través de la construcción, en lugar de la instrucción. Por lo tanto, su innovación pedagógica se enfocó por igual en el aprendizaje de la lengua extranjera y en el desarrollo de contenidos. En su estudio, a los estudiantes se les proporcionó la posibilidad de expresarse verbal y no verbalmente, y a participar en una variedad de actividades creativas en un contexto de enseñanza multimodal. Los hallazgos de su proyecto evidenciaron mejoras en los estudiantes, relacionadas con sus habilidades receptivas y productivas en el idioma, el desarrollo de la consciencia de ciudadanía y su sensibilidad hacia la diversidad.

Por último, los estándares nacionales para el aprendizaje de lenguas extranjeras- en contraste con los estándares de cualquier otra disciplina- muestran una gama muy limitada de las competencias lingüísticas e insisten en centrarse en la gramática de la lengua. Este énfasis en el aprendizaje de la lengua y no en el lenguaje como tal, no se conecta de manera eficaz con la vida y la realidad de los alumnos que necesitan utilizar el inglés para participar plenamente como ciudadanos críticos.

\section{Referencias}

Cárdenas, R. (2009). Tendencias globales y locales en la formación de docentes de lenguas extranjeras. Íkala, revista de lenguaje y cultura, 14(22), 71-105.

Coyle, D., Hood, P., \& Marsh, D. (2010). CLIL: Content and language integrated learning. Cambridge: Cambridge University Press.

Korosidou E., \& Griva, E. (2016). "It's the same world through different eyes": A content and language integrated learning project for young EFL learners. Colombian Applied Linguistics Journal, 18(1), pp 116-132.

Amparo Clavijo Olarte PhD

Editora 